Härle/Popp/Runte (Hrsg.) • Ikonen des Begehrens 
Gerhard Härle/Wolfgang Popp/Annette Runte (Hrsg.)

\section{Ikonen des Begehrens}

Bildsprachen der männlichen und weiblichen Homosexualität in Literatur und Kunst 
Für finanzielle Unterstützung des Kolloquiums und dieser Publikation danken wir:

- der Deutschen Forschungs-Gemeinschaft (DFG)

- der Gesellschaft der Freunde und Förderer der Universität-GH Siegen e.V.

- dem Kanzler der Universität-GH Siegen

- der Gesellschaft zur Förderung literarwissenschaftlicher Homostudien (GflH)

- der August von Platen Stiftung Siegen

-u.a.

Die Deutsche Bibliothek - CIP-Einheitsaufnahme

Ikonen des Begehrens:

Gerhard Härle/Wolfgang Popp/Annette Runte (Hrsg.)

- Stuttgart : M und P, Verl. für Wiss. und Forschung, 1997

ISBN 978-3-476-45173-6

\section{ISBN 978-3-476-45173-6}

ISBN 978-3-476-04268-2 (eBook)

DOI 10.1007/978-3-476-04268-2

Dieses Werk ist einschließlich aller seiner Teile geschützt. Jede Verwertung außerhalb der engen Grenzen des Urheberrechtsgesetzes ist ohne Zustimmung des Verlages unzulässig und strafbar. Das gilt insbesondere für die Vervielfältigungen, Übersetzung, Mikroverfilmungen und Einspeicherung in elektronischen Systemen.

M \& P Verlag für Wissenschaft und Forschung ein Verlag der J.B. Metzlerschen Verlagsbuchhandlung und Carl Ernst Poeschel Verlag GmbH in Stuttgart

(C) 1997 Springer-Verlag GmbH Deutschland Ursprünglich erschienen bei J.B. Metzlersche Verlagsbuchhandlung und Carl Ernst Poeschel Verlag GmbH in Stuttgart 1997 


\section{Inhalt}

Vorbemerkung

7

\section{Grundlagen}

GERHARD HÄRLE

Die Anwesenheit des eigentlich Abwesenden

Versuch über Erotik und Ikonologie

MARITA KEILSON-LAURITZ

Ganymed trifft Tadzio

Überlegungen zu einem 'Kanon der Gestalten'

JULIKA FUNK

„Butch \& Femme“. Original oder Kopie?

Ver-Führung zu einer lesbischen Ikonologie

\section{Zu einzelnen Ikonen in Literatur und Kunst}

WOLFGANG POPP

Der biblische David als schwule Ikone

der Kunst und Literatur

MeChTHILd FEND

Nebulöse Identitäten

Girodets Schlaf des Endymion

ULRIKE BERGERMANN

Das Bildnis der Gertrude Stein

Picassos Portrait im Blick der Queer Theory

Claudia ÖHLSCHLÄGER

"Die Erscheinung der Gewalt ist seltsam schön“

Caravaggio als Ikone tödlicher Obsession

bei Christoph Meckel und Christoph Geiser 


\section{Zu Ikonen in Film und Kunst}

Víctor R. CASTRO-Gómez

Eros und Gewalt

Die Figur Sebastians als Leitmotiv

homoerotischer Ikonographie (Mishima, Jarman, Williams)

Claudia Reiche

"Think pink and the world is rosy-red“"

Versuch einer Lektüre zwischen den Bildern

des Films Funny Face von Stanley Donen

DiRK NAgUSCHEWSKI

Arabische Jungen/ junge Araber

Mit Cadinot auf den Spuren Gides

\section{Camp-Kultur}

ULLA LINK-HEER

Robert de Montesquiou

Vom ikonisierten Leben zur Literatur

oder das Problem der Preziosität

Annette Runte

Camp as pop can

Andy Warhol als Gesamtkunstwerk

DIRCK LINCK

Der Hipster Irving Rosenthal:

„Everything is itch and scratch"

Ralph J. Poole

Bisse statt Küsse

Der 'queer' Vampir als Ikone des Camp

Abbildungsnachweise 379

Autorinnen und Autoren des Bandes 382 


\section{Vorbemerkung}

Der vorliegende Band versammelt 14 Beiträge, die während des 7. Siegener Kolloquiums Homosexualität und Literatur vom 12. bis 15. Oktober 1995 vorgetragen und diskutiert wurden. Mit dieser Tagung setzten die Veranstalter eine Reihe von entsprechenden internationalen Symposien fort, die seit über zehn Jahren im Forschungsgebiet Homosexualität und Literatur der Universität-Gesamthochschule Siegen durchgeführt werden. Sie ermöglichen überregional und international die Diskussion von Forschungsergebnissen zum Zusammenhang zwischen Literatur und Homosexualität und wollen Impulse für neue Forschungsansätze zu diesem Themengebiet geben. In diesem Sinne versuchen die Fachtagungen jeweils herausragende und/oder umstrittene Problemstellungen so $\mathrm{zu}$ focussieren, daß sowohl Aspekte der männlichen und der weiblichen Homosexualität und Homosozialität zum tragen kommen als auch interdisziplinäre Zugänge eröffnet werden. So standen etwa beim 3. Kolloquium 1991 mit dem Titel „Erkenntniswunsch und Diskretion“ Grundfragen des Zusammenhangs von erotischer Disposition und (auto-)biographischem Schreiben zur Diskussion. ${ }^{1} 1993$ war das 5. Kolloquium dem brisanten und durch die Krankheit AIDS überaus aktuellen Thema „Homosexualität und Krankheit" gewidmet, wobei auch hier die Diskussion über diesen „prekären Zusammenhang“ sowohl historische und sozialwissenschaftliche als auch kunsttheoretische Akzente setzte. ${ }^{2}$

Das 7. Siegener Kolloquium stand unter dem Thema „Ikonen des Begehrens. Bildsprachen der männlichen und weiblichen Homosexualität in Literatur und Kunst" und folgte wie die vorhergehenden Siegener Kolloquien der Intention, männliche und weibliche Homosexualität als ein Phänomen zu

1 Die Beiträge sind veröffentlicht in: Gerhard Härle, Maria Kalveram und Wolfgang Popp (Hg.): Erkenntniswunsch und Diskretion. Erotik in biographischer und autobiographischer Literatur. Berlin: rosa Winkel 1992 [Homosexualität und Literatur Band 6].

2 Die Beiträge dieser Tagung wurden veröffentlicht in den Heften der Zeitschrift Forum Homosexualität und Literatur: 18/1993, 19/1993, 20/1994 und 23/1995. 
untersuchen, das gleichermaßen seit jeher die europäische Kunst und Kultur entscheidend beeinflußt und zugleich als existentieller Faktor unzählige einzelne Lebensschicksale prägt. Die Grundproblematik der Bildsprache des Begehrens läßt sich im Bereich des Literarischen und der Literaturwissenschaft als die Frage nach der Wirksamkeit von Intertextualität festmachen, im weiteren kulturellen Zusammenhang als die Frage nach dem Vorhandensein und der Wirkung von Interdiskursen der Körperwahrnehmung und des Körperbegehrens. Das bedeutete auch für diese Tagung, daß sich wissenschaftliche Auseinandersetzung als ethisch und gesellschaftlich verantwortlich erweisen und in einem der Realität verpflichteten Bezugsrahmen stattfinden muß.

Über die Thematik im engeren Sinne hinaus war das Kolloquium auch dem Gedenken Oscar Wilde's gewidmet, weil dieser Autor der viktorianischen Epoche die Ikonologie des homosexuellen Begehrens wie kaum ein zweiter beeinflußt hat. Sein Roman Das Bildnis des Dorian Gray entwirft geradezu exemplarisch die Leistungsfähigkeit und die Grenzen einer ikonographischen Bildsprache in der Literatur, die das verbotene oder diffamierte erotische Begehren - Selbstliebe als Chiffre der Homosexualität - zu gestalten versucht. Hinzu kommt, daß der vor 100 Jahren, im Jahr 1895 von Oscar Wilde angestrengte Strafprozeß in seiner Bedeutung für die Geschichte der Homosexualität noch kaum erfaßt ist. Die Tatsache, daß Wilde durch diesen Prozeß wegen seiner Homosexualität vom Gipfel seines Ruhms und gesellschaftlichen Ansehens in den Abgrund einer existenzvernichtenden Strafgefangenschaft stürzte, hatte für die Homosexuellen in ganz Europa Auswirkungen, die mit den Auswirkungen der Dreyfus-Affäre auf die französische Judenschaft vergleichbar sind: einerseits wirkte das wider alle Erwartung an Wilde vollstreckte Urteil als abschreckendes Exempel auf zahllose ,gewöhnliche Homosexuelle"; andererseits zog gerade dieses Urteil in seiner irrationalen Härte auch Gegenbewegungen nach sich und wurde auf diese Weise zu einem der auslösenden Faktoren für die erste deutsche Schwulenbewegung, die bis zu ihrer Zerstörung durch die Nationalsozialisten ein wichtiger Faktor der deutschen Kultur des ersten Jahrhundertdrittels war. 
Als die Verantwortlichen für die Tagung und für die Herausgabe dieses Bandes danken wir allen Beiträgerinnen und Beiträgern, durch deren Vorträge die Diskussion angeregt und vorangetrieben wurde und die uns ihre Texte mit bereitwilliger Selbstlosigkeit überlassen haben. Unser Dank gilt auch Ilse Hennecke, Katja Höllriegel, Dr. Dirck Linck, Detlev Siniauvsky und Henning Pingel für ihre stets hilfreiche Mitarbeit beim Kolloquium und bei der Herstellung der Druckvorlagen, den Verlagen und Galerien, die uns die Rechte an den Bildmaterialien überlassen haben sowie der Deutschen Forschungsgemeinschaft (DFG), dem Kanzler, dem Graduierten-Kolleg und der Gesellschaft der Freunde und Förderer der Universität-Gesamthochschule Siegen, der Gesellschaft zur Förderung literarwissenschaftlicher Homostudien (GFlH) und der August von Platen-Stiftung für die finanzielle und ideelle Förderung der Tagung und der Publikation.

Nicht alle Referate standen für den Sammelband zur Verfügung; einige - vor allem aus dem Kreis der Oscar-Wilde-Thematik - sind bereits in der Zeitschrift Forum Homosexualität und Literatur veröffentlicht worden. ${ }^{3}$ Die hier versammelten Texte stehen jedoch repräsentativ ein für die Positionen und Diskussionen, für die Standpunkte und für die Bewegungen, die dem Kolloquium „Ikonen des Begehrens“ seinen unverwechselbaren Charakter gegeben haben und die auch über seine Grenzen hinaus ihre Wirkung entfalten können.

Siegen, im März 1997

Gerhard Härle, Annette Runte, Wolfgang Popp

3 Heft 25/1996: Cecilia Dreymüller: Göttin der Zweideutigkeit. Erotische Ikonen in der Lyrik von Christina Peri Rossi (S. 55-64); Horst Weich: Der Jüngling, der Gott. die Statue: Luis Antonio Villenas Ikonen des Begehrens (S. 65-86).

Heft 26/1996: Wolfram Setz: Die Geburt des Homosexuellen aus dem Geiste der Literatur. Anmerkungen zum Prozeß gegen Oscar Wilde (S. 7-26); Hans Richard Brittnacher: „Der Geck war tragisch“. Hofmanntshals Nachruf auf Oscar Wilde (S. 27-42); Ernest W. B. Hess-Lüttich: Dandy, Camp und fin du globe. Wildes Inversion viktorianischer Werte (S. 43-68). 